\section{International Journal of Medical Research and Review}

\title{
The correlation of doxorubicin related cardiotoxicity with B-type natriuretic peptide levels in South Indian population
}

\author{
Koshy Jacob R. ${ }^{1}$, V. Karpurmath S. ${ }^{2 *}$, Nandennavar M. ${ }^{3}$, Angadi V. ${ }^{4}$ \\ DOI: https://doi.org/10.17511/ijmrr.2019.i06.03 \\ 1 Roshan Koshy Jacob, Department of Medical Oncology, Vydehi Institute of Medical Sciences \& Research Centre, Bengaluru, Karnataka, \\ 2* Shashidhar V. Karpurmath, Department of Medical Oncology, Vydehi Institute of Medical Sciences \& Research Centre, Bengaluru, \\ 3 Manjunath Nandennavar, Department of Medical Oncology, Vydehi Institute of Medical Sciences \& Research Centre, Bengaluru, Karnataka, \\ ${ }^{4}$ Veerendra Angadi, Department of Medical Oncology, Vydehi Institute of Medical Sciences \& Research Centre, Bengaluru, Karnataka, India.
} India. Karnataka, India. India.

Background: Anthracyclines represent the greatest risk for development of cardiotoxicity. Cardiotoxicity of anthracyclines may develop during the treatment (acute cardiotoxicity) and during the follow-up (chronic and late cardiotoxicity). Natriuretic peptides - Atrial Natriuretic Peptide (ANP), B-type Natriuretic Peptide (BNP) and N-terminal pro B-type natriuretic peptide (NT-pro-BNP) are released by myocardium in response to wall strain and pressure overload. Aims and Objectives: To observe correlation of NT-pro-BNP levels with cardiotoxicity in patients receiving doxorubicin. Methods and Materials: Eighty patients who were planned for treatment with Doxorubicin $>200$ $\mathrm{mg} / \mathrm{m}^{2}$ were included in this study. Each patient was assessed clinically (History, Pulse rate, Blood pressure) along with ECG, ECHO and NT-pro-BNP levels prior to initiation of chemotherapy, after completion of $200 \mathrm{mg} / \mathrm{m}^{2}$ of Doxorubicin, 3 months and 6 months after chemotherapy. Result: There were total of 80 patients in the study and they received a total of 384 cycles of Doxorubicin containing regimens according to respective protocols. The median number of cycles was four (range four to six cycles). The mean cumulative dose of doxorubicin was $267.75 \mathrm{mg} / \mathrm{m}^{2}$. Conclusion: Based on the findings in this study it can be concluded that high upfront BNP values or increasing values of BNP does not correlate with the incidence of acute and early onset chronic cardiotoxicity.

Keywords: Doxorubicin, NT-pro-BNP, Cardiotoxicity, ECHO

\section{Corresponding Author}

Shashidhar V. Karpurmath, Department of Medical Oncology, Vydehi Institute of Medical Sciences \& Research Centre, Bengaluru, Karnataka, India. Email: shashivk5@gmail.com
How to Cite this Article To Browse Roshan Koshy Jacob, Shashidhar V. Karpurmath, Manjunath Nandennavar, Veerendra Angadi, The correlation of doxorubicin related cardiotoxicity with B-type natriuretic peptide levels in South Indian population. Int J Med Res Rev. 2019;7(6):452-460. Available From

https://ijmrr.medresearch.in/index.php/ijmrr/article/

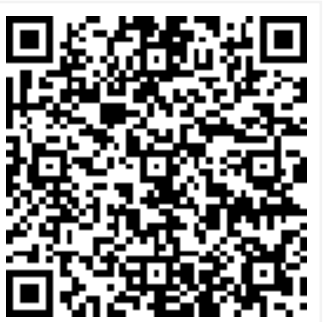
view/1094
Manuscript Received 2019-10-14

Conflict of Interest No

Review Round 1
2019-10-24
Funding
Nil

Tunding

(C) 2019 by Roshan Koshy Jacob, Shashidhar V. Karpurmath, Manjunath Nandennavar, Veerendra Angadi and Published by Siddharth Health Research and Social Welfare Society. This is an Open Access article licensed under a Creative Commons Attribution 4.0 International License https://creativecommons.org/licenses/by/4.0/ unported [CC BY 4.0]

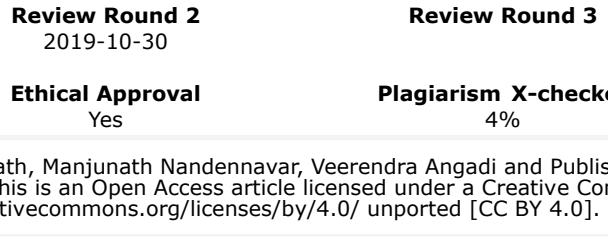




\section{Introduction}

Anthracyclines represent the greatest risk for development of cardiotoxicity. The most common manifestations of anthracycline cardiotoxicity are arrhythmias, cardiac dysfunction (cardiomyopathy, heart failure), pericardial injury and myocardial ischemia. Cardiotoxicity of anthracyclines may develop during the treatment (acute cardiotoxicity) and during the follow-up (chronic and late cardiotoxicity). Clinical efficacy is often limited by associated cardiotoxicity leading to cardiomyopathy that may worsen the patient's outcome [1].

According to the time of onset, cardiotoxicities are classified into:

1) "Acute", occurring after a single dose, or a single course, of anthracyclines, with onset of clinical manifestations within two weeks from the end of treatment;

2) "Early onset chronic", developing within 6 months -1 year. This is the most frequent and clinically relevant form of cardiotoxicity, usually presenting as a dilated and hypokinetic cardiomyopathy leading to heart failure (HF);

3) "Late onset chronic" developing years, or even decades, after the end of chemotherapy [2].

The total cumulative dose is one of the main risk factor for anthracycline-related cardiotoxicity. However, there is no completely safe dose, and so the cardiotoxicity of any given dosage must always be weighed against efficacy of the drug. Treatment duration may also influence the risk of cardiotoxicity during or after therapy and prolonged administration have shown to reduce the severity of cardiac injury. Younger patients are more vulnerable to the cardiotoxic effect of anthracyclines [3].

Various methods have been recommended for monitoring of cardiotoxicity in oncology including Echocardiography (ECHO) and electrocardiography (ECG) which are routinely used. Serial non-invasive surveillance for evidence of cardiotoxicity in patients receiving anthracyclines has centered on the Echocardiographic assessment of left ventricular (LV) systolic function using ejection-phase indices, namely, fractional shortening and ejection fraction. LV shortening fraction and ejection fraction have shown to be valuable clinical predictors of morbidity and mortality in children and adults with malignancies who receive anthracyclines as part of their therapeutic regimen [4].
Biochemical markers of cardiac injury, especially natriuretic peptides and cardiac troponins, have been studied in this anthracycline-induced cardiotoxicity. Natriuretic peptides - Atrial Natriuretic Peptide (ANP), B-type Natriuretic Peptide (BNP) and $\mathrm{N}$-terminal pro $\mathrm{B}$-type natriuretic peptide (NT-pro-BNP) are released by myocardium in response to wall strain and pressure overload. Natriuretic peptides levels also increase in subjects with acute and chronic cardiac dysfunction and heart failure.

As such, testing for natriuretic peptides levels has been recognized and incorporated in the guidelines for diagnosis and management of heart failure. The applicability of natriuretic peptides (ANP, BNP, NTpro-BNP) as markers for Anthracy cline-induced cardiotoxicity has been investigated in a few studies.

The results from these studies suggest that Natriuretic peptides could be of value in the detection of clinical and subclinical cardiotoxicity of Anthracyclines as well as markers of cardiac toxicity after high-dose chemotherapy and hematopoietic stem cell transplantation [1].

There are no major studies done in our population regarding the correlation of anthracycline induced cardiotoxicity with BNP levels. All previous trials have used higher dose of anthracyclines and there are no studies to assess the incidence of cardiotoxicity in lesser doses of anthracyclines (200$400 \mathrm{mg} / \mathrm{m} 2$ ).

The present study has been undertaken to correlate the anthracycline induced cardiotoxicity and BNP levels in South Indian population.

\section{Methods}

\section{Source of Data}

- The source of data for the study is patients with any malignancy presenting to the Department of Medical Oncology, Vydehi Institute of Medical Sciences, Bangalore who will receive Doxorubicin $>200 \mathrm{mg} / \mathrm{m} 2$ as part of treatment protocol.

- Duration of study - January 2016 to June 2017

- Study Design- Interventional

- Type of study - Prospective Study

- Sampling Size - 80. The Sample size was estimated in consultation with a biostatistician. 
- Ethical committee- Approved

\section{Method of collection of data (including sampling, procedure, if any)}

- A standard proforma will be used to collect patient related data for the study.

- Informed consent will be taken.

- Patients who will receive Doxorubicin $>200$ $\mathrm{mg} / \mathrm{m} 2$ as part of treatment protocol will be randomly allocated to the study. Demographic details of these patients will be entered in the proforma. Each patient was assessed clinically (History, Pulse rate, Blood pressure) along with ECG, ECHO and NT-pro BNP prior to initiation of chemotherapy, after completion of $200 \mathrm{mg} / \mathrm{m} 2$ of Doxorubicin, 3 months and 6 months after chemotherapy.

- Patients were observed for developments of signs and symptoms of CHF, cardiomyopathy characterized by decrease in the LVEF that was either global or more severe in the septum, decline in the LVEF of at least $5 \%$ to less than $55 \%$ with signs or symptoms of $\mathrm{CHF}$, or a decline in LVEF of at least $10 \%$, to below $55 \%$ without signs or symptoms which would suggest cardiotoxicity.

\section{Inclusion criteria}

- Age more than 18 years and less than 70 years.

- Patients with any malignancy who will receive > $200 \mathrm{mg} / \mathrm{m} 2$ of Doxorubicin as part of the treatment protocol.

\section{Exclusion criteria}

- History of valvular or coronary cardiac diseases.

- Prior exposure to anthracyclines.

- Prior exposure to irradiation to the mediastinum.

- Patient with LVEF $<55 \%$ in the baseline ECHO

Statistical analysis- The Statistical software namely SPSS 18.0, and R environment ver.3.2.2 were used for the analysis of the data and Microsoft word and Excel have been used to generate graphs, tables etc.

Statistical methods: Descriptive and inferential statistical analysis has been carried out in the present study.

Results on continuous measurements are presented on Mean \pm SD (Min-Max) and results on categorical measurements are presented in Number (\%). Significance is assessed at $5 \%$ level of significance.
The following assumptions on data is made

01. Dependent variables should be normally distributed

02. Samples drawn from the population should be random, Cases of the samples should be independent

Student $\mathrm{t}$ test (two tailed, dependent) has been used to find the significance of study parameters on continuous scale. Paired Proportion test has been used to find the significance of proportion in paired data. Smaller percentage of Improvement becomes significant at lower tail compared to higher tail. E.g. Improvement from $10 \%$ to $20 \%$ is difficult than the Improvement from $80 \%$ to $90 \%$ [5-8].

\section{Results}

A total number of 80 patients with any malignancy presenting to the Department of Medical Oncology, Vydehi Institute of Medical Sciences, Bangalore who received Doxorubicin $>200 \mathrm{mg} / \mathrm{m2}$ as part of treatment protocol were recruited from January 2016 to June 2017 for this study. The patients were selected according to the inclusion and exclusion criteria as mentioned earlier. Before including the patient in the study, a detailed consent was taken from the patient.

All demographic details were noted in the proforma along with investigations carried out initially and those repeated as mentioned earlier. 80 patients received a total of 384 cycles of Doxorubicin containing regimens according to respective protocols. Each patient was assessed clinically (History, Pulse rate, Blood pressure) along with ECG,ECHO and NT-pro-BNP levels prior to initiation of chemotherapy, after completion of $200 \mathrm{mg} / \mathrm{m} 2$ of Doxorubicin, 3 months and 6 months after chemotherapy.

In the present study the age range of patients included was between 18 years and 70 years of age. The mean age of the patients was 44.33 years. Data on the age distribution of patients included in the study is shown in Table 1 . Most of the patients were between the age group of 31 years to 50 years. A total of 57 females and 23 males were included in the study as depicted in Table 2.

Table-1: Age distribution of patients studied.

\begin{tabular}{|l|l|l|}
\hline \multicolumn{1}{|c|}{ Age in years } & \multicolumn{1}{|c|}{ No. of patients } & \multicolumn{1}{c|}{$\%$} \\
\hline $20-30$ & 15 & 18.8 \\
\hline $31-40$ & 14 & 17.5 \\
\hline $41-50$ & 27 & 33.8 \\
\hline
\end{tabular}


Jacob R.K. et al: The correlation of doxorubicin related

\begin{tabular}{|l|l|l|}
\hline $51-60$ & 16 & 20.0 \\
\hline $61-70$ & 8 & 10.0 \\
\hline Total & 80 & 100.0 \\
\hline
\end{tabular}

Mean \pm SD: $44.33 \pm 11.93$

Table-2: Gender distribution of patients studied.

\begin{tabular}{|l|l|l|}
\hline \multicolumn{1}{|c|}{ Gender } & \multicolumn{1}{c|}{ No. of patients } & \multicolumn{1}{c|}{$\%$} \\
\hline Female & 57 & 71.3 \\
\hline Male & 23 & 28.8 \\
\hline Total & 80 & 100.0 \\
\hline
\end{tabular}

Among the study population of 80 patients, only 11 patients had history of co-morbid conditions which included 6 hypertensive patients, 4 patients with diabetes mellitus and one patient with Congenital heart Disease- Atrial Septal Defect- Post surgical closure. All these patients were on regular medication for these respective illnesses. Table 3 gives the BSA distribution of the study population. Average height was $157.16 \mathrm{~cm}$ and average weight was $55.91 \mathrm{~kg}$ in the study population of 80 patients.

Table-3: BSA- distribution of patients studied.

\begin{tabular}{|l|l|l|}
\hline \multicolumn{1}{|c|}{ BSA } & \multicolumn{1}{c|}{ No. of patients } & \multicolumn{1}{c|}{$\%$} \\
\hline$<1.5$ & 35 & 43.8 \\
\hline $1.5-1.8$ & 40 & 50.0 \\
\hline$>1.8$ & 5 & 6.3 \\
\hline Total & 80 & 100.0 \\
\hline
\end{tabular}

In the present study majority of the patients were Stage III accounting to almost $62.5 \%$ of the total study population. There were around 19 patients with Stage IV disease which was $23.8 \%$. The Stage distribution of the study population is mentioned in Table 4.

Table-4: Stage of disease distribution of patients studied.

\begin{tabular}{|l|l|l|}
\hline \multicolumn{1}{|c|}{ Stage } & \multicolumn{1}{c|}{ No. of patients } & \multicolumn{1}{c|}{$\%$} \\
\hline I & 1 & 1.3 \\
\hline II & 10 & 12.5 \\
\hline III & 50 & 62.5 \\
\hline IV & 19 & 23.8 \\
\hline Total & 80 & 100.0 \\
\hline
\end{tabular}

In the present study the maximum numbers of patients were carcinoma breast patients constituting almost $60 \%$ of the total study population. The next common diagnosis was Non Hodgkins Lymphoma. The details regarding the diagnosis distribution is mentioned below in Table 5 .

Table-5: Diagnosis- distribution of patients studied.

\begin{tabular}{|l|l|l|}
\hline \multicolumn{1}{|c|}{ Diagnosis } & No. of patients & \multicolumn{1}{c|}{$\%$} \\
\hline Carcinoma Breast & 48 & 60.0 \\
\hline Non-Hodgkins Lymphoma & 21 & 26.3 \\
\hline Hodgkins Lymphoma & 5 & 6.3 \\
\hline Soft tissue sarcoma & 4 & 5.0 \\
\hline Osteosarcoma & 1 & 1.3 \\
\hline Phyloides tumour Breast & 1 & 1.3 \\
\hline Total & 80 & 100.0 \\
\hline
\end{tabular}

Details regarding the Doxorubicin containing chemotherapy regimens used are mentioned below in Table 6.

Table-6: Chemo regimen distribution of patients studied.

\begin{tabular}{|l|l|l|}
\hline \multicolumn{1}{|c|}{ Chemo regimen } & \multicolumn{1}{c|}{ No. of patients } & \multicolumn{1}{c|}{$\%$} \\
\hline AC & 48 & 60.0 \\
\hline R-CHOP & 15 & 18.8 \\
\hline ABVD & 5 & 6.3 \\
\hline IA & 5 & 6.3 \\
\hline CHOP & 4 & 5.0 \\
\hline CHOP-E & 2 & 2.5 \\
\hline IAP & 1 & 1.3 \\
\hline Total & 80 & 100.0 \\
\hline
\end{tabular}

Since majority of the patients included in the present study were carcinoma breast patients, the most used chemotherapy regimen was AC (Doxorubicin and Cyclophosphamide). The median number of cycles is four (range four to six cycles) as seen in Table 7.

Table-7: No. of cycles distribution of patients studied.

\begin{tabular}{|l|l|l|}
\hline \multicolumn{1}{|c|}{ No. of cycles } & \multicolumn{1}{c|}{ No. of patients } & \multicolumn{1}{c|}{$\%$} \\
\hline 4 & 48 & 60.0 \\
\hline 6 & 32 & 40.0 \\
\hline Total & 80 & 100.0 \\
\hline
\end{tabular}

As shown in Table 8, the mean cumulative dose of doxorubicin was $267.75 \mathrm{mg} / \mathrm{m} 2$. All patients received full course of chemotherapy as planned upfront. Each patient was clinically assessed (History, Pulse rate and Blood pressure) along with ECG, ECHO and NT-pro-BNP prior to initiation of chemotherapy, after completion of $200 \mathrm{mg} / \mathrm{m} 2,3$ months and 6 months after chemotherapy.

Table- 8: Dose of Doxorubicin received $\mathrm{mg} / \mathrm{mz}$.

\begin{tabular}{|l|l|l|}
\hline Dose of Doxorubicin received $\mathbf{~ m g / m 2}$ & No. of patients & \multicolumn{1}{c|}{$\%$} \\
\hline 240 & 48 & 60.0 \\
\hline 300 & 27 & 33.8 \\
\hline 360 & 5 & 6.3 \\
\hline Total & 80 & 100.0 \\
\hline
\end{tabular}


None of the patients developed any cardiac symptoms during or after the planned chemotherapy. There was no drop in Left Ventricular Ejection Fraction on serial ECHO during the period of the study. Total of 4 patients had upfront BNP values $>300 \mathrm{pg} / \mathrm{ml}$ and 4 patients with moderate elevation of NT-pro-BNP (200-300 pg/ml), however none of these patients developed acute or early chronic cardiotoxicity.

14 patients had serially increasing values of NT-proBNP in the 6 months follow-up, however none of these patients developed acute or early chronic cardiotoxicity. Whether these patients will develop late chronic cardiotoxicity needs to be confirmed with a longer follow-up. NT-pro-BNP distribution and mean values are mentioned in Table 9 and Table 10.

Table 9: BNP (pg/ml)- Assessment from baseline at different study time points of patients studied.

\begin{tabular}{|l|l|l|l|l|}
\hline \begin{tabular}{|l|l|} 
BNP \\
$(\mathrm{pg} / \mathrm{ml})$
\end{tabular} & Upfront & $\begin{array}{r}\text { After } 200 \\
\mathrm{mg} / \mathrm{m} 2 \text { of } \\
\text { Doxorubicin }\end{array}$ & $\begin{array}{l}3 \text { months after } \\
\text { chemotherapy }\end{array}$ & $\begin{array}{l}6 \text { months after } \\
\text { chemotherapy }\end{array}$ \\
\hline $1-50$ & $43(53.8 \%$ & $24(30 \%)$ & $15(18.8 \%)$ & $17(21.3 \%)$ \\
\hline $51-100$ & $15(18.8 \%$ & $20(25 \%)$ & $22(27.5 \%)$ & $31(38.8 \%)$ \\
\hline $101-200$ & $14(17.5 \%$ & $18(22.5 \%)$ & $23(28.8 \%)$ & $26(32.5 \%)$ \\
\hline $201-500$ & $5(6.3 \%)$ & $16(20 \%)$ & $19(23.8 \%)$ & $5(6.3 \%)$ \\
\hline$>500$ & $3(3.8 \%)$ & $2(2.5 \%)$ & $1(1.3 \%)$ & $1(1.3 \%)$ \\
\hline Total & $80(100 \%$ & $80(100 \%)$ & $80(100 \%)$ & $80(100 \%)$ \\
\hline
\end{tabular}

Table-10: BNP (pg/ml)- Comparison from baseline at different study time points of patients studied

\begin{tabular}{|l|l|l|}
\hline \multicolumn{1}{|c|}{ BNP $(\mathrm{pg} / \mathrm{ml})$} & \multicolumn{1}{c|}{ Min-Max } & \multicolumn{1}{c|}{ Mean \pm SD } \\
\hline Upfront & $5.00-2233.00$ & $116.59 \pm 278.48$ \\
\hline After $200 \mathrm{mg} / \mathrm{m} 2$ of Doxorubicin & $18.00-1233.00$ & $140.00-164.61$ \\
\hline 3 months after chemotherapy & $22.00-855.00$ & $145.43 \pm 125.44$ \\
\hline 6 months after chemotherapy & $18.00-610.00$ & $105.40 \pm 79.07$ \\
\hline
\end{tabular}

\section{Discussion}

Anthracyclines are among the most commonly used chemotherapy drugs. However, their efficacy is sidelined by potential life-threatening cardiotoxicity. Anthracy-cline induced cardiotoxicity is potentiated when the cumulative dose of doxorubicin exceeds $300 \mathrm{mg} / \mathrm{m} 2$. Symptomatic heart failure is rare with cumulative dose of $240 \mathrm{mg} / \mathrm{m} 2$ [9].
Natriuretic peptides have been demonstrated to be useful markers of LV dysfunction in both symptomatic and asymptomatic patients. High concentrations of plasma natriuretic peptides have been described not only in patients with acute myocardial infarction or advanced CHF but also in patients with asymptomatic or minimally symptomatic LV dysfunction. Only a few published reports have evaluated the role of natriuretic peptides in chemotherapy-treated patients. Some studies concluded that there is an association between LV dysfunction and increased natriuretic peptide concentrations [10].

In the present study 80 patients who presented to our institute with malignancy and who were planned for Doxorubicin containing chemotherapy regimens $>200 \mathrm{mg} / \mathrm{m} 2$ were included in the study. However, in study conducted by Sandri et al, retrospectively 52 patients treated with High Dose Chemotherapy for aggressive malignancy from January 2000 to December 2000 and from July 2002 to August 2003were included in the study. In the present study, the mean age of the total study population was 44.33 years with majority if patients being in the age group 41 to 50 years. Patients above the age of 60 years accounted only for $10 \%$ of the study population. In comparison the study done by Sandri et al had mean age of 47 years.

In the present study there were total of 57 females and 23 males distributed evenly in the 2 groups. Compared to this, the study done by Sandri et al had 17 men and 35 women. Majority of the patients in the present study had a BSA ranging from 1.41.8. This accounted for almost $74 \%$ of the total study population. The mean height of the study population was $157.16 \mathrm{~cm}$ and the mean weight was $55.91 \mathrm{~kg}$.

In the present study had 48 patients with carcinoma breast, 21 patients with Non-Hodgkins lymphoma and few patients with Hodgkins Lymphoma, soft tissue sarcoma, osteosarcoma and one patient with Phylloides tumour breast. Stage III and Stage IV patients constituted almost $86 \%$ of the study population in the present study. On the other hand, in the study done by Sandri et al, there were 52 patients with aggressive malignancy who underwent high dose chemotherapy followed by autologous stem cell transplantation.

The chemotherapy regimens used in the present study included AC, R-CHOP, CHOP, CHOP-E, ABVD, IA and IAP. 
Among these regimens, $A C$ regimen was the most commonly used regimen as majority of study population were cases of Carcinoma breast. In the present study median number of cycles was 4 , with 48 patients receiving 4 cycles of chemotherapy and the rest receiving 6 cycles.

Sandri et al used High dose chemotherapy every 28 days for 1-3 cycles depending on the specific chemotherapy schedule. All patients underwent reinfusion of autologous peripheral blood progenitor cells during each course of High dose chemotherapy.

The mean cumulative dose of doxorubicin was $267.75 \mathrm{mg} / \mathrm{m} 2$ in the present study. Only 5 out of the 80 patients received doses more than 300 $\mathrm{mg} / \mathrm{m} 2$. Among the study population of 80 patients, only 11 patients had history of co-morbid conditions which included 6 hypertensive patients, 4 patients with diabetes mellitus and one patient with Congenital Heart disease - Atrial Septal Defect Post surgical closure. All these patients were on regular medication for these respective illnesses.

None of the patients in the present study developed any cardiac symptoms or a drop in the Left Ventricular Ejection Fraction or shortening fraction on serial ECHO during the period of the study. As none of the patients in the study developed cardiotoxicity, correlation between ECG changes and NT-pro-BNP values could not be done.

However in the study done by Sandri et al, seventeen patients $(33 \%)$ had persistently increased NT-pro-BNP, 19 patients (36\%) had only transient increases (concentrations went back to baseline at $72 \mathrm{~h})$, and $16(31 \%)$ had no increases [mean (SD) values at $72 \mathrm{~h}, 1163$ (936) ng/L vs 185 (101) $\mathrm{ng} / \mathrm{L}$ vs 39 (19) $\mathrm{ng} / \mathrm{L}$, respectively; $\mathrm{P}$ $<0.0001]$.

Only patients with persistently increased NT-proBNP had a significant worsening of the left ventricular diastolic indexes from baseline to 12 months [ratio of peak early to peak late flow velocities from $1.42(0.33)$ to 0.78 (0.11)] isovolumetric relaxation time from 90 and of the left ventricular ejection fraction [from 62.8 to 45.6] [10]

In one study done by Horacek et al, twenty-six adult patients (mean age $46.2 \pm 12.4$ years, 15 males) were studied. They were treated with 2-6 cycles of chemotherapy (CT) containing anthracyclines in the total cumulative dose of $464.3 \pm 117.5 \mathrm{mg} / \mathrm{m} 2$. Cardiac evaluation with biochemical markers - " $\mathrm{N}$ terminal pro brain natriuretic peptide" (NT-pro-
BNP), cardiac troponin $\mathrm{T}$ (cTnT), creatine kinase MB (CK-MB), and echocardiography was performed at baseline, after first and last $\mathrm{CT}$ with anthracyclines and 6 months after CT. Mean baseline NT-pro-BNP concentration was $117.7 \pm 46.4 \mathrm{ng} / \mathrm{L}$ (slightly elevated in 3 patients).

After first and last chemotherapy, NT-pro-BNP elevations to $299.7 \pm 176.2 \mathrm{ng} / \mathrm{L}$ and $287.1 \pm 147.4$ $\mathrm{ng} / \mathrm{L}$ were observed, respectively. Six months after chemotherapy, mean NT-pro-BNP concentration was $362.5 \pm 304.9 \mathrm{ng} / \mathrm{L}$ (elevated in 16 patients). Changes in NT-pro-BNP were significant in comparison with the baseline values $(p<0.001)$. Six months after chemotherapy, two patients with marked NT-pro-BNP elevations during CT developed treatment-related cardiomyopathy with symptoms of heart failure. NT-pro-BNP correlated with systolic and diastolic LV dysfunction on echocardiography ( $r$ $=0.514 ; p<0.01)$ and $(r=0.587 ; p<0.01)$. Cardiac Troponin $\mathrm{T}$ concentrations were negative (bellow $0.01 \mu \mathrm{g} / \mathrm{L}$ ) during chemotherapy in all patients.

The study concluded that anthracycline treatment is associated with acute and chronic neurohumoral activation of cardiac dysfunction that is manifested by a significant increase in NT-pro-BNP. NT-pro-BNP could be useful in the early detection of anthracycline cardiotoxicity. Cardiac Troponin $\mathrm{T}$ negativity during anthracycline treatment suggests that anthracyclines, even in higher cumulative doses, do not cause detectable acute injury to cardiomyocyte structure [1].

A total of 8 independent case-control studies, containing 126 anthracycline-induced cardiotoxicity patients and 569 healthy controls, were included for the current meta analysis. The results indicated a significant difference in serum BNP levels between the cardiotoxic group and normal group, with respect to post-treatment and pre-treatment with anthracyclines.

Specifically, the serum levels of BNP increased remarkably after treatment with anthracyclines in the cardiotoxic group, compared with the normal group. The findings of the present study provide strong evidence that serum BNP levels may be associated with anthracycline-induced cardiotoxicity. [11].

In a study done by Romano et al, 71 patients undergoing Not High Dose Chemotherapy (NHDC) with anthracyclines were evaluated with NT-pro-BNP and cardiac Troponin I levels measured before and 
24h after each NHDC cycle along with Left ventricular (LV) function was assessed by echocardiography at baseline, every two NHDC cycles, at the end of chemotherapy, and at 3-, 6and 12-month follow-up. According to NT-pro-BNP behaviour, patients were divided into two groups with group A (n-50) with normal (n-23) or transiently elevated NT-pro-BNP levels ( $n-27)$, and group B (n-21) with persistently elevated NT-proBNP levels.

At 12-month follow-up, two patients experienced dyspnoea (after the end of chemotherapy) with clinical evidence of heart failure, and two additional patients developed supraventricular arrhythmias (during chemotherapy). All these four patients belonged to group B (with persistent NT-pro-BNP elevation). Hence the author concluded that serial measurements of NT-pro-BNP may be a useful tool for the early detection of patients treated with NHDC at high risk of developing cardiotoxicity [12].

In the present study, as none of the patients developed cardiotoxicity, correlation with NT-proBNP levels was not possible. There were 4 patients who had very high values of NT-pro-BNP ( $>300$ $\mathrm{pg} / \mathrm{ml}$ ) and 4 patients with moderate elevation of NT-pro-BNP $(200-300 \mathrm{pg} / \mathrm{ml})$ prior to the initiation of chemotherapy. 14 patients had serially increasing values of NT-pro-BNP in the 6 months follow-up. However there was no development of acute or early chronic cardiotoxicity in any of these patients during the period of the study.

Hence it can be safely concluded that upfront elevated NT-pro-BNP or serially raising values of NTpro-BNP cannot be taken as a marker for development of acute or early chronic cardiotoxicity associated with adminis-tration of doxorubicin. The correlation of NT-pro-BNP with late chronic cardiotoxicity cannot be commented in this study in view of the short follow-up of 6 months.

\section{Limitations of this Study}

01. Patients were followed up only for a period of 6 months. Patients are being kept on longer follow-up of upto 3 years to watch for development of late chronic cardiotoxicity and the updated result will be published in due course of time. If patient develops late chronic cardiotoxicity during follow-up, then the correlation with upfront and serial NT-pro-BNP values can be done.
02. The number of patients included in the study is small $(n=80)$

03. The dose of Doxorubicin used in this study population was less compared to those studies in which patients developed doxorubicin induced cardiotoxicity as most of the newer chemotherapy protocol limit the dose of doxorubicin.

\section{Conclusion}

Upfront elevated NT-pro-BNP or serially raising values of NT-pro-BNP cannot be taken as a marker for development of acute or early chronic cardiotoxicity associated with administration of doxorubicin. The correlation of NT-pro-BNP with late chronic cardiotoxicity cannot be commented in this study in view of the short follow-up of 6 months. These patients will be kept of longer follow-up to watch for development of late chronic cardiotoxicity due to Doxorubicin and the updated result will be published in due course of time.

\section{What study adds to the existing knowledge?}

There are conflicting results about the role of NTpro-BNP in predicting Doxorubicin related cardiotoxicity. Some studies have shown a correlation between the two while some studies have not shown a correlation.

Also there is scarcity of data in this aspect in the Indian Population. With this study being done in Indian population, it can be concluded that there is no correlation of elevated NT-pro-NP values or serially rising NT-pro-BNP values with Doxorubicin related acute or early chronic cardiotoxicity in our population. Whether or not NT-pro-BNP correlates with late chronic cardiotoxicity can be concluded only with longer follow-up of these patients.

\section{Author's contributions}

Dr. Roshan Koshy Jacob: Concept, study design, manuscript preparation.

Dr. Shashidhar V. Karpurmath: Analysis of the data, manuscript preparation.

Dr. Manjunath Nandennavar: Patients follow-up.

Dr. Veerendra Angadi: Patients follow-up. 


\section{Acknowledgements}

Author take this opportunity to thank a number of people who have been an integral part in the completion of this study. Author would like to offer utmost and heart-felt thanks and respect to professor, teacher and guide Dr. Shashidhar V.K, Professor and Head of department of Medical Oncology, Vydehi Institute of Medical Sciences and Research Centre, Bangalore, for lending his constant support, encouragement, motivation and invaluable guidance during the preparation of the manuscript and for analysis of the data.

Author is thankful to Dr. Manjunath I $\mathrm{N}$ and Dr. Veerendra for their support in times of need or otherwise. They helped in the follow-up of the 80 patients and also for getting required investigations during the follow-up period. Author would like to profusely thank all the colleagues for unflinchingly sharing the work and providing help which was much needed. Author would also like to extend gratitude towards radiation oncology and surgical oncology colleagues for their support in times of need. Lastly author would like to thank all the patients who have given consent to be a part of this study and without whom it would not have been possible.

\section{Reference}

01. Horacek JM, Pudil R, Jebavy L, Tichy M, Zak P, Maly J. Assessment of anthracycline-induced cardiotoxicity with biochemical markers. Exp Oncol. 2007;29(4)309-313.

[Crossref]

02. Cardinale D, Colombo A, Bacchiani G, Tedeschi I, Meroni CA, Veglia $F$, et al. Early detection of anthracycline cardiotoxicity and improvement with heart failure therapy. Circulat. 2015;131

(22)1981-88. doi: 10.1161/CIRCULATIONAHA.114.01 3777 [Crossref]

03. Adão R, de Keulenaer G, Leite-Moreira A, BrásSilva C. Cardiotoxicity associated with cancer therapy- patho-physiology and prevention strat egies. Rev Port Cardiol. 2013;32(5)395-409. doi: 10.1016/j.repc.2012.11.002 [Crossref]

04. Eidem BW. Identification of anthracycline cardiotoxicity: left ventricular ejection fraction is not enough. J Am Soc Echocardiogr. 2008;21(12)1290-1292.

doi: $\quad 10.1016 /$ j.echo.2008.10.008 [Crossref]
05. Rosner B. Fundamentals of Biostatistics. 5th Ed Duxbury. 2010;80-240.

[Crossref]

06. Robert H. Statistics in Medicine. 2nd edition, Academic press. 2005;85-125.

[Crossref]

07. Rao PS, Richard J. An Introduction to Biostatistics- A manual for students in health sciences. Prentice/Hall of India. 1996.

[Crossref]

08. Suresh KP, Chandrasekhar S. Sample Size estimation and Power analysis for Clinical research studies. J Human Reproduct Sci. 2012; 5(1)7-13.

doi: $10.4103 / 0974-1208.97779$ [Crossref]

09. Hershman DL, Shao T. Anthracycline cardiotoxicity after breast cancer treatment. Oncol (Williston Park). 2009;23(3)227-234. [Crossref]

10. Sandri MT, Salvatici M, Cardinale D, Zorzino L, Passerini R, Lentati P. N-terminal pro-B-type natriuretic peptide after high-dose chemotherapy- a marker predictive of cardiac dysfunction?. Clin Chem. 2005;51(8)14051410.

doi: 10. 1373/clinchem.2005.050153 [Crossref]

11. Wang YD, Chen SX, Ren LQ. Serum B-type natriuretic peptide levels as a marker for anthracycline-induced cardiotoxicity. Oncol Let. 2016;11(5)3483-3492.

[Crossref]

12. Romano $S$, Fratini $S$, Ricevuto $E$, Procaccini $V$, Stifano $G$, Mancini $M$, et al. Serial measurements of NT-proBNP are predictive of not-high-dose anthracycline cardiotoxicity in breast cancer patients. Brit J Cancer. 2011;105(11)1663-1668.

doi: 10.1038/bjc.2011.439 [Crossref] 